\title{
Linkage disequilibrium mapping as an advancement in crop breeding
}

\section{Introduction}

Linkage disequilibrium refers to the non random association of alleles between genetic loci on the same or different chromosome. It is also called as gametic phase disequilibrium. LD occurs when genotypes at the two loci are not independent of another. LD is the result of physical linkage of genes. LD could be the outcome of recent migration, recent selection, and new mutation and increased by self pollination, inbreeding, low recombination rate, and genetic isolation between lineage, population admixture, population subdivision and epistasis. While factors like out-crossing, high recombination rate, high mutation rate and gene conversion leads to decrease or decay in linkage disequilibrium. ${ }^{1,2}$

Association mapping or linkage disequilibrium mapping is a method of mapping quantitative trait loci (QTLs) using historical meiotic recombination events performed over several generations to link phenotypes (observable characteristics) with genotypes (genetic constitution) in large germplasm populations. It is a powerful genetic mapping tool for crops and provides high-resolution, broad allele coverage, and cost-effective gene tagging for the evaluation of plant germplasm resources.

\section{Concept of linkage disequilibrium mapping}

Association mapping is based on the principle that over several generations of recombination, correlations of linked markers with trait of interest will remain. Therefore, spurious associations between genotype and trait may be detected due to the degree of structure within the population, necessitating development of various statistical methods to account for population structure., ${ }^{2,3}$ Association mapping give surety of high resolution mapping by exploitation of historical recombination events at the population level that may enable gene level mapping on non-model organisms where linkage-based approaches would not be feasible. Potential exploit of such approach could be for fine mapping of genes/QTLs, identifying favorable alleles for marker assisted selection and cross validation of outcome from linkage mapping for accurate position of genes/QTLs of interest. ${ }^{4}$

\section{Genetic mapping of QTLs}

Genetic mapping of QTLs can be performed in two main ways:

i. Linkage analysis based mapping or conventional mapping using experimental populations or bi-parental mapping populations and

ii. Linkage disequilibrium based mapping or "association mapping" using diverse lines from the natural populations or germplasm collections or landraces. Linkage based QTL mapping can identify the related genes to biparental variations so that mapping resolution depends on the number of recombinations occurred in the process of the development of mapping populations. Furthermore, construction of a suitable mapping population for study is very time consuming. Association mapping has recently become popular for identifying and mapping QTLs with high resolution. Linkage disequilibrium (LD) based association mapping can lead
Volume 6 Issue 2 - 2017

\author{
Parmeshwar Kumar Sahu, Deepak Sharma \\ Department of Genetics and Plant Breeding, India
}

Correspondence: Parmeshwar Kumar Sahu, Department of Genetics and Plant Breeding, IGKV, Raipur- 492012 (Chhattisgarh), India, Email parmeshwarsahu1210@gmail.com

Received: September 05, 2017 | Published: January 30, 2017

to the most effective utilization of ex-situ conserved natural genetic diversity or germplasm resources of crop plants.

\section{Advantages of linkage disequilibrium mapping over conventional linkage mapping}

LD mapping has many major advantages over linkage analysis based QTL mapping

i. Much larger and more representative gene pool can be surveyed

ii. Much higher resolution can be achieved

iii. Greater number of allelic diversity can be analyzed

iv. No need to generate bi-parental mapping population so research time will be less

$v$. It enables the mapping of many traits in one set of genotypes

vi. Finally, it has the potential not only to identify and map QTLs but also to identify the causal polymorphism within a gene that is responsible for the difference in two alternative phenotypes. ${ }^{4,5}$

vii. Unlike family-based linkage analysis, LD mapping does not require family or pedigree information and can be applied to a range of experimental and non-experimental populations.

\section{Computer Soft ware's for analysis}

There are several free and paid softwares available for association mapping analysis. Some of them are as follows, TASSEL, STRUCTURE, EMMA, SAS, R, PLINK, BAPS, GenStat, JMP Genomics, FaST-LMM, GGT, MIDAS, SVS7 etc.

\section{Acknowledgements}

None.

\section{Conflict of interest}

The author declares no conflict of interest.

\section{References}

1. Yu J, Buckler ES. Genetic association mapping and genome organization of maize. Current Opinion in Biotechnology. 2006;17(2):155-160. 
2. Vinod KK. Association mapping in crop plants. Advanced faculty training on "impact of genomics on crop improvement: perceived and achieved" TNAU, Coimbatore; 2011.

3. Balding DJ. A tutorial on statistical methods for population association studies. Natural Genetics. 2006;7:781-791.
4. Rosyara UR, Joshi BK. Association mapping for Improvement of Quantitative traits in Plant breeding population. Nepal journal of biotechnology. 2012;2(1):72-89.

5. Sehgal D, Singh R, Rajpal VR. Quantitative trait loci mapping in plants: concepts and approaches. Molecular Breeding for Sustainable Crop Improvement Springer International Publishing Switzerland; 2016. p. $31-59$. 